\title{
La influencia de las diferencias entre el padre y la madre sobre el ajuste adolescente
}

\author{
Antonia Jiménez-Iglesias* y Carmen Moreno
}

Departamento de Psicología Evolutiva y de la Educación, Universidad de Sevilla

\begin{abstract}
Resumen: Este trabajo se centra en analizar cómo influyen en el consumo de sustancias (tabaco, alcohol y cannabis) y el bienestar psicológico adolescente (calidad de vida relacionada con la salud y satisfacción vital) las diferencias entre el padre y la madre en afecto, promoción de la autonomía, revelación, interés y conocimiento. La muestra estuvo compuesta por 10170 chicos y chicas españoles de 13 a 18 años de familias biparentales que participaron en la edición 2006 del estudio Health Behaviour in School-aged Children (HBSC). Los resultados mostraron que los adolescentes que indicaron que su padre y madre coincidían en presentar altos niveles en las dimensiones familiares analizadas consumieron menos sustancias y tuvieron mejor bienestar psicológico. Por el contrario, el ajuste de los adolescentes cuyos progenitores (padre y madre) tenían bajos niveles en esas dimensiones familiares fue peor. Concretamente, la dimensión más importante para el consumo de sustancias fue el conocimiento parental y para el bienestar psicológico lo fue el afecto parental; la revelación adolescente solo fue relevante para el consumo de alcohol y la calidad de vida, mientras que la promoción parental de la autonomía lo fue para la calidad de vida.

Palabras clave: Dimensiones familiares; padres y madres; adolescencia; consumo de sustancias; bienestar psicológico.
\end{abstract}

\section{Introducción}

Las investigaciones que analizan la relación entre el estilo parental y el ajuste adolescente suelen diferenciar entre el enfoque tipológico y el enfoque dimensional. El enfoque dimensional considera la influencia de variables maternas y paternas concretas del estilo parental (como el afecto, el control o la comunicación) sobre el desarrollo. Por su parte, el enfoque tipológico tiene en cuenta la relación entre estas dimensiones para clasificar a los progenitores según su estilo educativo en democráticos, permisivos, autoritarios e indiferentes (Steinberg y Silk, 2002).

La investigación desde el enfoque tipológico nos muestra que lo más común en las familias es encontrar que ambos progenitores tengan el mismo estilo parental (Casullo y Liporace, 2008; Gamble, Ramakumar y Diaz, 2007; Simons y Conger, 2007), pero también padres y madres pueden ser diferentes en ello. Las diferencias entre los estilos maternos y paternos han recibido poca atención (McKinney y Renk, 2008), dado que habitualmente solo se han clasificado los maternos o se han evaluado los estilos parentales de padres y madres, pero se han excluido del análisis a las familias en las que los progenitores mostraban diferentes estilos, o bien se han promediado las puntuaciones de padres y madres, lo que puede llevar a clasificar mal a las familias y a no conocer las consecuencias reales para los hijos y las hijas (Simons y Conger, 2007).

En relación con las consecuencias que las similitudes o divergencias entre el padre y la madre pueden tener para los

* Dirección para correspondencia [Correspondence address]: Antonia Jiménez-Iglesias. Departamento de Psicología Evolutiva y de la Educación. C/ Camilo José Cela, s/n. 41018 Sevilla (España). E-mail: amjimigl@us.es
Title: The influence of differences between father and mother on adolescent adjustment.

Abstract: This paper analyzes how the differences between father and mother in affection, promotion of autonomy, disclosure, solicitation and knowledge influence on substance use (tobacco, alcohol and cannabis) and psychological well-being (health-related quality of life and life satisfaction). The sample was composed of 10170 boys and girls aged 13 to 18 years from two-parent families who participated in 2006 edition of Health Behaviour in School-aged Children (HBSC) study. Results indicated that adolescents whose both parents had high values for the different family dimensions used less substance and scored higher in psychological well-being. In contrast, the adjustment of adolescents whose both parents had low values for the different family dimensions was worse. Specifically, the most important dimension for substance use was parental knowledge and for psychological well-being was parental affection; adolescent disclosure was only relevant for alcohol use and quality of life, and parental promotion of autonomy for quality of life.

Key words: Family dimensions; parents; adolescence; substance use; psychological well-being.

hijos y las hijas, parece que un estilo parental consistente en ambos progenitores es más efectivo y deseable que si es inconsistente (Dwairy, 2010). Sin embargo, a pesar de que durante la infancia la coherencia entre las prácticas y los estilos educativos de padres y madres resulta fundamental (Steinberg, 2001), en la adolescencia esta coherencia o consistencia entre los progenitores resulta menos relevante que las ventajas que puede suponer disponer de, al menos, un progenitor democrático (Fletcher, Steinberg y Sellers, 1999; McKinney y Renk, 2008; Steinberg, 2001).

De este modo, los resultados más positivos para los adolescentes se consiguen cuando los dos progenitores son democráticos (McKinney y Renk, 2008; Simons y Conger, 2007; Steinberg, 2001). Pero, en la ausencia de este estilo parental óptimo, tener un progenitor de este tipo puede, en la mayoría de los casos, amortiguar al chico o la chica de las consecuencias perjudiciales asociadas con un menos óptimo estilo parental (Simons y Conger, 2007), ya que la diferencia existente entre el ajuste psicológico y comportamental de los chicos y las chicas con dos progenitores democráticos y el de quienes solo tienen uno, es bastante menor que la diferencia entre quienes tienen, al menos, un progenitor democrático y quienes no tienen ninguno (Steinberg, 2001).

Los peores resultados se encuentran cuando los dos progenitores son indiferentes. Cuando solo uno de los progenitores muestra este estilo, las combinaciones que incluyen una madre indiferente producen significativamente peores resultados que aquellas combinaciones en las que el padre lo es (Simons y Conger, 2007), lo cual indica que la influencia materna suele ser algo superior que la paterna (Hair, Moore, Garrett, Ling y Cleveland, 2008; Rodrigo et al., 2004).

$\mathrm{Al}$ analizar las diferencias entre progenitores desde un enfoque dimensional se hallan datos similares a los obteni- 
dos según el enfoque tipológico. Concretamente, al estudiar la influencia de la facilidad y la dificultad de la comunicación con el padre y la madre en el consumo de sustancias, se encontró que los adolescentes con un menor consumo de sustancias pertenecieron al grupo que decía tener mayor facilidad para la comunicación con ambos progenitores, seguido del grupo que dijo tener facilidad sólo con la madre; tras ambos grupos, los adolescentes que más consumieron fueron quienes indicaron tener facilidad para comunicarse sólo con el padre y quienes no la tuvieron con ninguno de los progenitores (Moreno, Muñoz-Tinoco, Pérez Moreno y Sánchez-Queija, 2006).

Esta investigación no se realiza teniendo en cuenta el enfoque tipológico, sino según el enfoque dimensional, ya que analiza determinadas dimensiones del estilo democrático que son componentes importantes del mismo (afecto parental, promoción parental de la autonomía, interés parental, revelación adolescente y conocimiento parental) y su influencia sobre el ajuste adolescente.

Las variables de ajuste adolescente que se examinan en este trabajo son el consumo de sustancias y el bienestar psicológico porque el desarrollo de las mismas en la adolescencia puede tener importantes implicaciones en la adultez. La adolescencia es la etapa más adecuada para evitar o cambiar cualquier hábito poco saludable, como es el consumo de sustancias, antes de que pueda ver su continuidad en la etapa evolutiva siguiente (Elliot 1993). Igualmente, el bienestar psicológico en la adolescencia es la base para la salud y el bienestar psicológico en la adultez (Williams, Holmbeck y Neff Greenley, 2002).

En relación con las dimensiones familiares analizadas, resulta necesario comentar su relevancia para el ajuste de chicos y chicas. Así, el afecto parental es una dimensión clave del estilo democrático y decisiva para el bienestar psicológico adolescente (Kagitçibasi, 1996), así como favorece un menor consumo de sustancias (Parra y Oliva, 2006; Rodrigo et al., 2004). Por otro lado, la promoción parental de la autonomía adquiere especial relevancia en la adolescencia; los padres y las madres que son sensibles a las nuevas necesidades de autonomía de sus adolescentes y la promueven de una forma adecuada mejoran su bienestar (Gillison, Standage y Skevington, 2008; Lekes, Gingras, Philippe, Koestner y Fang, 2010; Smetana, Campione-Barr y Daddis, 2004) y reducen su consumo de sustancias (Goldstein, Davis-Kean y Eccles, 2005). De igual manera, la revelación adolescente y el interés parental implican la existencia de una positiva comunicación parento-filial (Keijsers, Branje, VanderValk y Meeus, 2010), que es protectora del consumo de sustancias y del bienestar psicológico (Jiménez-Iglesias, Moreno, Granado-Alcón y López, 2012).

Finalmente, el conocimiento parental, la información que disponen los padres y las madres de sus hijos e hijas, es producto de un positivo funcionamiento familiar, cercano y comunicativo, como el que supone el estilo democrático (Bumpus y Rodgers, 2009). Este conocimiento permite a los padres y madres estar informados de las vidas de sus hijos e hijas y usar ese conocimiento para protegerles de conductas de riesgo, como el consumo de sustancias (Stattin, Kerr y Tilton-Weaver, 2010), así como promover efectivamente el bienestar adolescente (Bronstein et al., 1996; Reitz, Deković y Meijer, 2006).

El objetivo de este trabajo radica en conocer qué dimensiones familiares (afecto, promoción de la autonomía, interés, revelación adolescente y conocimiento parental) son más relevantes para el consumo de sustancias (concretamente se analizan el consumo de tabaco, de alcohol y de cannabis) y el bienestar psicológico (específicamente son examinadas la calidad de vida relacionada con la salud y la satisfacción vital) de los adolescentes cuando dichas dimensiones se presentan en niveles altos y bajos en ambos progenitores, o en combinaciones de niveles altos y bajos en el padre y la madre.

\section{Método}

\section{Participantes}

Los participantes procedieron de familias biparentales de la edición 2006 del estudio internacional Health Behaviour in School-aged Children (HBSC). En total fueron 12324 adolescentes, pero para este trabajo solo se seleccionaron a quienes respondieron a todas las variables que se analizan, en concreto a 10170 adolescentes españoles (45\% chicos y 55\% chicas) de 13 a 18 años $(M=15.90, D T=1.56)$. Más información acerca del procedimiento de muestreo puede ser encontrada en Moreno et al. (2011).

\section{Instrumentos}

El cuestionario HBSC de la edición 2006 superó los requisitos exigidos por el Comité Ético de Experimentación de la Universidad de Sevilla para la experimentación en sujetos humanos y en animales, ajustándose a la normativa vigente en España y en la Unión Europea.

Las dimensiones familiares analizadas fueron las siguientes: afecto materno y paterno, escalas basadas en la dimensión de afecto del Parental Bonding Inventory-Brief Current form (PBI-BC) de Klimidis, Minas y Ata, 1992, y que obtuvieron en este trabajo valores del alfa de Cronbach de .74 para el afecto materno y .81 para el afecto paterno. Promoción materna y paterna de la autonomía, escalas basadas en la dimensión de promoción de la autonomía del PBI-BC de Klimidis et al., 1992, cuyos valores del alfa de Cronbach en este trabajo fueron de .60 para la dimensión materna y .58 para la paterna. Interés materno y paterno y revelación del adolescente a la madre y al padre, cada dimensión fue medida con dos ítems, uno para el padre y otro para la madre. Conocimiento materno y paterno, escalas tomadas del instrumento construido por Brown, Mounts, Lamborn y Steinberg (1993); los valores de fiabilidad para este trabajo fueron de.74 para el conocimiento materno y de .82 para el conocimiento paterno. Todas estas dimensiones fueron transformadas en categóricas, para este trabajo, y tuvieron un total de cuatro ca- 
tegorías cada una: bajo madre-bajo padre, bajo madre-alto padre, alto madre-bajo padre, alto madre-alto padre.

Las variables de ajuste adolescente fueron consumo de sustancias y bienestar psicológico.

En relación con el consumo de sustancias, se analizaron la frecuencia de consumo de tabaco semanal (Hublet y Godeau, 2005), con valores desde 0 (nunca) a 7 (todos los días); la máxima frecuencia de consumo de bebidas alcohólicas durante la semana (Schmid, Fotiou, Godeau, Simons-Morton y Hublet, 2005), igualmente con valores desde 0 (nunca) a 7 (todos los días); y la frecuencia de consumo de cannabis en los últimos 12 meses, con valores desde 0 (nunca) a 40 (40 veces o más), adaptada del European School Survey Project on Alcohol and Other Drugs, ESPAD (Hibell et al., 2000).

En cuanto al bienestar psicológico se examinó la calidad de vida relacionada con la salud, medida de forma cuantitativa con media 50, desviación típica 10, y con alfa de Cronbach de .80 en este trabajo; se trata del cuestionario KIDSCREEN-10 index (The KIDSCREEN Group Europe, 2006). Además de la satisfacción vital, con valores de 0 (la peor vida posible) a 10 (la mejor vida posible), basada en la conocida Escalera de Cantril (Cantril, 1965).

\section{Procedimientos}

En el procedimiento de recogida de información, el organismo internacional del estudio HBSC señala que deben cumplirse tres condiciones básicas. En primer lugar, los propios escolares deben de ser quienes respondan al cuestionario; en segundo lugar, se ha de asegurar y respetar escrupulosamente el anonimato de las respuestas; y, por último, la administración de los cuestionarios debe realizarse dentro del contexto escolar por encuestadores entrenados (Currie et al., 2008).

En los análisis estadísticos, el programa utilizado fue el IBM SPSS Statistics 18. Para el objetivo de este trabajo se realizaron ANOVAs factoriales con el procedimiento modelo lineal general univariante usando modelos de efectos principales. La prueba de significación usada fue la $F$ y el índice de tamaño del efecto para el modelo general fue la $R^{2}$, que ha sido calificada como despreciable de 0 a .019 , de .02 a .129 como baja, de .13 a .259 como media y como grande a partir de .26. Para cada variable concreta, el índice de tamaño del efecto ha sido la eta cuadrado parcial, considerando los siguientes intervalos: de 0 a .009, despreciable, de .01 a .059 supone un tamaño de efecto bajo, de .06 a .149 indica un tamaño de efecto medio y a partir de .15 un tamaño de efecto grande (Cohen, 1988). Si este análisis resultó significativo y el tamaño de efecto apreciable, se efectuaron comparaciones múltiples post hoc mediante la prueba de Bonferroni, para conocer dónde se encuentran las diferencias entre las medias en cada variable (Shaughnessy, Zechmeister y Zechmeister, 2007); en las comparaciones de dos medias, el tamaño de efecto usado ha sido la $d$ de Cohen según los siguientes crite- rios: de 0 a 0.19 indica un efecto despreciable; de 0.20 a 0.49 un tamaño de efecto bajo; de 0.50 a 0.79 señala un tamaño de efecto medio; y a partir de 0.80 un tamaño de efecto grande (Cohen, 1988).

\section{Resultados}

\section{Consumo de sustancias}

En la Tabla 1 se presentan los datos descriptivos del consumo de sustancias en función del afecto parental, la promoción parental de la autonomía, el interés parental, la revelación adolescente y el conocimiento parental. En general, los valores medios de consumo de tabaco, alcohol y cannabis fueron más altos cuando ambos progenitores puntuaron bajo en las dimensiones familiares y más bajos cuando ambos progenitores puntuaron alto en las mismas, salvo para el consumo de alcohol y de cannabis según la promoción parental de la autonomía (en los que las diferencias no fueron tan claras).

En relación con el consumo de tabaco, la Tabla 2 muestra que el modelo resultó significativo y con tamaño de efecto bajo, $F(15,9361)=27.851, R^{2}=.043$. Los resultados fueron significativos para el interés parental, $F(3,9361)=$ $6.574, p<.001$ y la revelación adolescente, $F(3,9361)=$ $11.338, p<.001$, pero los tamaños de efecto fueron despreciables $\left(\eta^{2}\right.$ parcial $=.002$ para interés y $\eta^{2}$ parcial $=.004$ para revelación). Sin embargo, para el conocimiento parental los resultados fueron significativos, $F(3,9361)=63.066, p<$ .001 , y con tamaño de efecto bajo $\left(\eta^{2}\right.$ parcial $\left.=.020\right)$.

Los adolescentes cuyos padres y madres puntuaron bajo en conocimiento, consumieron más tabaco que los adolescentes con madres poco conocedoras y padres muy conocedores $(p=.005, d=0.37)$, con madres y padres en la situación inversa $(p<.001, d=0.29)$ y, con una diferencia de magnitud más grande, con madres y padres con altos niveles de esta dimensión $(p<.001, d=0.73)$. Además, los adolescentes con ambos progenitores conocedores de sus experiencias consumieron menos tabaco que los adolescentes teniendo solo a las madres como muy conocedoras $(p<.001, d$ $=0.36)$. En la Figura 1 se representan estas diferencias.

El modelo para el consumo de alcohol resultó significativo y con tamaño de efecto bajo, $F(15,9361)=28.745, p<$ $.001, R^{2}=.044$, como se observa en la Tabla 3. La promoción parental de la autonomía fue significativa, $F(3,9361)=$ $6.647, p<.001$, pero con tamaño de efecto despreciable $\left(\eta^{2}\right.$ parcial $=.002)$. Sí tuvieron bajos tamaños de efecto $\left(\eta^{2}\right.$ parcial $=.012$ para revelación y $\eta^{2}$ parcial $=.015$ para conocimiento) y fueron significativos la revelación adolescente, $F$ $(3,9361)=39.392, p<.001, y$ el conocimiento parental, $F$ $(3,9361)=46.416, p<.001$. 
Tabla 1. Datos descriptivos del consumo de tabaco, alcohol y cannabis según las dimensiones familiares

\begin{tabular}{|c|c|c|c|c|c|c|c|c|c|c|}
\hline \multirow{2}{*}{ Variable } & & \multicolumn{3}{|c|}{ Consumo de tabaco } & \multicolumn{3}{|c|}{ Consumo de alcohol } & \multicolumn{3}{|c|}{ Consumo de cannabis } \\
\hline & & $N$ & $M$ & DT & $N$ & $M$ & $D T$ & $N$ & $M$ & $D T$ \\
\hline \multirow{4}{*}{ Afecto parental } & bajo madre-bajo padre & 836 & 1.62 & 2.73 & 836 & 1.26 & 1.72 & 836 & 4.88 & 10.74 \\
\hline & bajo madre-alto padre & 345 & 1.30 & 2.47 & 345 & 1.19 & 1.67 & 345 & 3.95 & 9.67 \\
\hline & alto madre-bajo padre & 1651 & 1.39 & 2.62 & 1651 & 1.18 & 1.52 & 1651 & 4.85 & 10.96 \\
\hline & alto madre-alto padre & 7337 & 0.98 & 2.28 & 7337 & 0.94 & 1.43 & 7337 & 3.07 & 9.09 \\
\hline \multirow{4}{*}{ Promoción parental de la autonomía } & bajo madre-bajo padre & 2251 & 1.30 & 2.54 & 2251 & 0.99 & 1.55 & 2251 & 3.53 & 9.43 \\
\hline & bajo madre-alto padre & 1392 & 1.11 & 2.37 & 1392 & 1.11 & 1.47 & 1392 & 3.71 & 9.56 \\
\hline & alto madre-bajo padre & 1028 & 1.22 & 2.47 & 1028 & 1.07 & 1.48 & 1028 & 3.52 & 9.27 \\
\hline & alto madre-alto padre & 5499 & 1.01 & 2.33 & 5499 & 0.98 & 1.47 & 5499 & 3.51 & 9.76 \\
\hline \multirow{4}{*}{ Interés parental } & bajo madre-bajo padre & 1242 & 1.49 & 2.63 & 1242 & 1.18 & 1.69 & 1242 & 4.68 & 11.38 \\
\hline & bajo madre-alto padre & 484 & 1.39 & 2.61 & 484 & 1.04 & 1.44 & 484 & 3.72 & 9.98 \\
\hline & alto madre-bajo padre & 2489 & 1.36 & 2.61 & 2489 & 1.13 & 1.48 & 2489 & 4.43 & 10.61 \\
\hline & alto madre-alto padre & 5955 & 0.90 & 2.20 & 5955 & 0.92 & 1.44 & 5955 & 2.92 & 8.64 \\
\hline \multirow{4}{*}{ Revelación adolescente } & bajo madre-bajo padre & 3781 & 1.46 & 2.63 & 3781 & 1.28 & 1.62 & 3781 & 5.15 & 11.46 \\
\hline & bajo madre-alto padre & 750 & 1.02 & 2.32 & 750 & 1.13 & 1.64 & 750 & 3.57 & 9.46 \\
\hline & alto madre-bajo padre & 1901 & 1.13 & 2.41 & 1901 & 0.99 & 1.42 & 1901 & 3.04 & 8.76 \\
\hline & alto madre-alto padre & 3738 & 0.77 & 2.09 & 3738 & 0.73 & 1.29 & 3738 & 2.17 & 7.54 \\
\hline \multirow{4}{*}{ Conocimiento parental } & bajo madre-bajo padre & 655 & 2.51 & 3.05 & 655 & 1.76 & 1.90 & 655 & 9.57 & 14.78 \\
\hline & bajo madre-alto padre & 69 & 1.39 & 2.48 & 69 & 1.93 & 2.16 & 69 & 7.48 & 13.43 \\
\hline & alto madre-bajo padre & 1647 & 1.68 & 2.80 & 1647 & 1.30 & 1.58 & 1647 & 5.39 & 11.41 \\
\hline & alto madre-alto padre & 7799 & 0.87 & 2.17 & 7799 & 0.88 & 1.39 & 7799 & 2.61 & 8.26 \\
\hline
\end{tabular}

Tabla 2. Modelo lineal general de las dimensiones familiares sobre el consumo de tabaco.

\begin{tabular}{|c|c|c|c|c|c|c|}
\hline Fuente & $S C$ & $g l$ & $M C$ & $F$ & $p$ & $\eta^{2}$ parcial \\
\hline Modelo corregido & 2341.303 & 15 & 156.087 & 27.851 & .000 & .043 \\
\hline Intersección & 1763.367 & 1 & 1763.367 & 314.648 & .000 & .033 \\
\hline Afecto parental & 7.675 & 3 & 2.558 & 0.456 & .713 & .000 \\
\hline Promoción parental de la autonomía & 33.892 & 3 & 11.297 & 2.016 & .109 & .001 \\
\hline Interés parental & 110.525 & 3 & 36.842 & 6.574 & .000 & .002 \\
\hline Revelación adolescente & 190.627 & 3 & 63.542 & 11.338 & .000 & .004 \\
\hline Conocimiento parental & 1060.315 & 3 & 353.438 & 63.066 & .000 & .020 \\
\hline Error & 52461.488 & 9361 & 5.604 & & & \\
\hline Total & 66774.000 & 9377 & & & & \\
\hline Total corregida & 54802.791 & 9376 & & & & \\
\hline
\end{tabular}

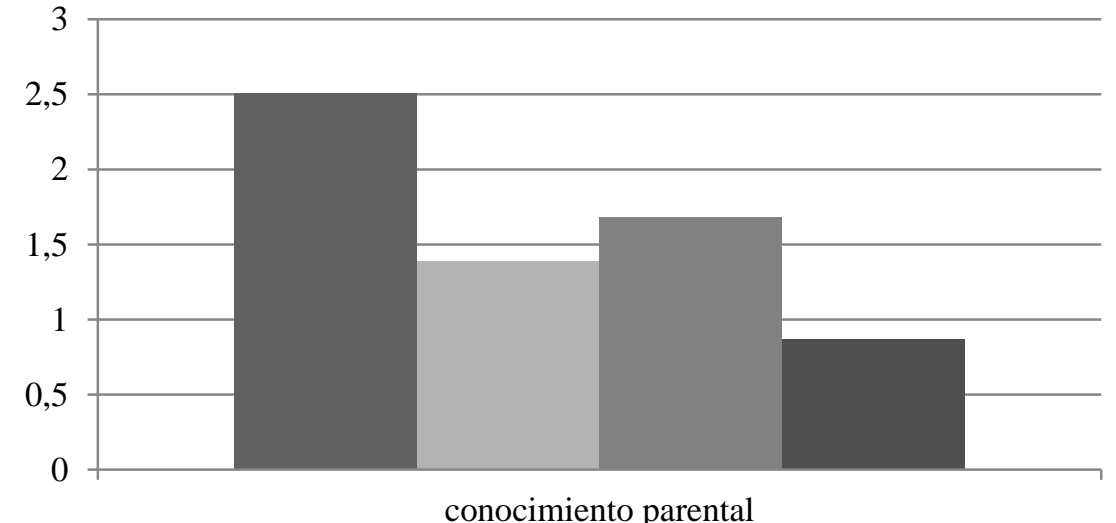

Figura 1. Consumo de tabaco según el conocimiento parental bajo madre-bajo padre

bajo madre-alto padre

alto madre-bajo padre

alto madre-alto padre 
Tabla 3. Modelo lineal general de las dimensiones familiares sobre el consumo de alcohol

\begin{tabular}{|c|c|c|c|c|c|c|}
\hline Fuente & $S C$ & $g l$ & $M C$ & $F$ & $p$ & $\eta^{2}$ parcial \\
\hline Modelo corregido & 920.305 & 15 & 61.354 & 28.745 & .000 & .044 \\
\hline Intersección & 1471.226 & 1 & 1471.226 & 689.295 & .000 & .069 \\
\hline Afecto parental & 2.656 & 3 & 0.885 & 0.415 & .742 & .000 \\
\hline Promoción parental de la autonomía & 42.564 & 3 & 14.188 & 6.647 & .000 & .002 \\
\hline Interés parental & 5.471 & 3 & 1.824 & 0.854 & .464 & .000 \\
\hline Revelación adolescente & 252.233 & 3 & 84.078 & 39.392 & .000 & .012 \\
\hline Conocimiento parental & 297.208 & 3 & 99.069 & 46.416 & .000 & .015 \\
\hline Error & 19980.035 & 9361 & 2.134 & & & \\
\hline Total & 30821.000 & 9377 & & & & \\
\hline Total corregida & 20900.340 & 9376 & & & & \\
\hline
\end{tabular}

Los adolescentes que informaron revelar a ambos progenitores $(p<.001, d=0.38)$ o solo a sus madres $(p<.001, d$ $=0.30)$ poca información, consumieron más alcohol que los adolescentes que revelaron mucha información a sus madres y padres. Además, cuando los progenitores eran percibidos como más conocedores de las experiencias de sus adolescentes, éstos consumieron menos alcohol que los adolescentes cuyos progenitores $(p<.001, d=0.62)$ y cuyas madres tuvie- ron bajo conocimiento $(p<.001, d=0.75)$, en ambos casos con magnitud del efecto medio, y cuyos padres puntuaron bajo en esta dimensión $(p<.001, d=0.30)$ con un tamaño de efecto pequeño. También, los adolescentes con un alto conocimiento materno percibido consumieron menos alcohol que los adolescentes con bajos conocimiento parental $(p<.001, d=0.28)$ y conocimiento materno $(p=.004, d=$ 0.39). La Figura 2 muestra estas diferencias.

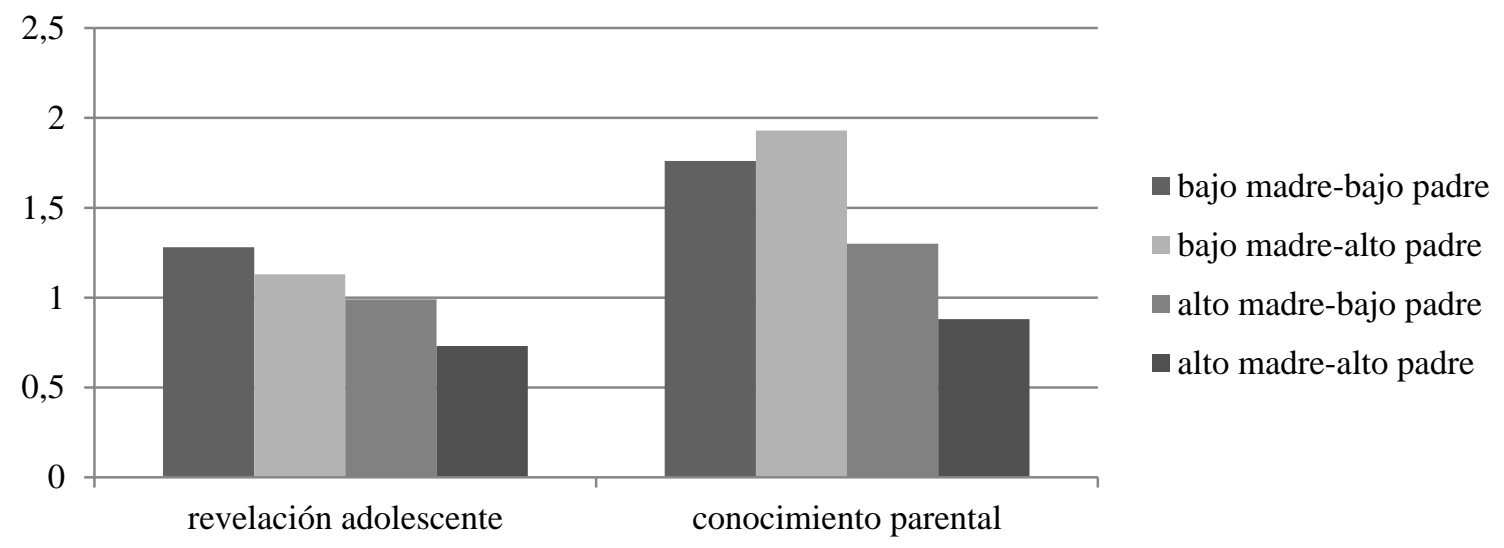

Figura 2. Consumo de alcohol según la revelación adolescente y el conocimiento parental

En cuanto al consumo de cannabis, como muestra la Tabla 4 , el modelo fue significativo y con tamaño de efecto bajo, $F(15,9361)=32.581, p<.001, R^{2}=.050$. La promoción parental de la autonomía, $F(3,9361)=6.824, p<.001$ y la revelación adolescente, $F(3,9361)=22.097, p<.001$, fue- ron significativas, pero con tamaños de efecto despreciables $\left(\eta^{2}\right.$ parcial $=.002$ para promoción de la autonomía y $\eta^{2}$ parcial $=.007$ para revelación). En cambio, el conocimiento parental fue significativo, $F(3,9361)=79.869, p<.001$, con bajo tamaño de efecto $\left(\eta^{2}\right.$ parcial $\left.=.025\right)$.

Tabla 4. Modelo lineal general de las dimensiones familiares sobre el consumo de cannabis

\begin{tabular}{|c|c|c|c|c|c|c|}
\hline Fuente & $S C$ & $g l$ & $M C$ & $F$ & $p$ & $\eta^{2}$ parcial \\
\hline Modelo corregido & 43864.947 & 15 & 2924.330 & 32.581 & .000 & .050 \\
\hline Intersección & 24477.026 & 1 & 24477.026 & 272.711 & .000 & .028 \\
\hline Afecto parental & 418.218 & 3 & 139.406 & 1.553 & 199 & .000 \\
\hline Promoción parental de la autonomía & 1837.530 & 3 & 612.510 & 6.824 & .000 & .002 \\
\hline Interés parental & 548.800 & 3 & 182.933 & 2.038 & .106 & .001 \\
\hline Revelación adolescente & 5949.991 & 3 & 1983.330 & 22.097 & .000 & .007 \\
\hline Conocimiento parental & 21505.796 & 3 & 7168.599 & 79.869 & .000 & .025 \\
\hline Error & 840190.359 & 9361 & 89.754 & & & \\
\hline Total & 1005904.000 & 9377 & & & & \\
\hline Total corregida & 884055.306 & 9376 & & & & \\
\hline
\end{tabular}


La diferencia con un efecto de magnitud cercano a grande estuvo entre los adolescentes cuyos progenitores fueron percibidos ambos con alto conocimiento y los adolescentes cuyos progenitores fueron valorados como menos conocedores, siendo estos últimos, los adolescentes que consumieron más cannabis $(p<.001, d=0.79)$. Diferencias de magnitud baja y media, respectivamente, se encontraron entre los adolescentes cuyos progenitores conocieron mucho sobre sus experiencias (quienes consumieron menos) y aquellos chicos y chicas cuyas madres conocieron mucho y sus padres poco $(p<.001, d=0.32)$ y los adolescentes en la situación inversa $(p<.001, d=0.59)$. Además, fueron más consumidores de cannabis aquellos adolescentes con ambos progenitores menos conocedores de sus experiencias que aquellos con padres que puntuaron bajo y madres que puntuaron alto en esta dimensión $(p<.001, d=0.34)$. La Figura 3 representa estas diferencias.

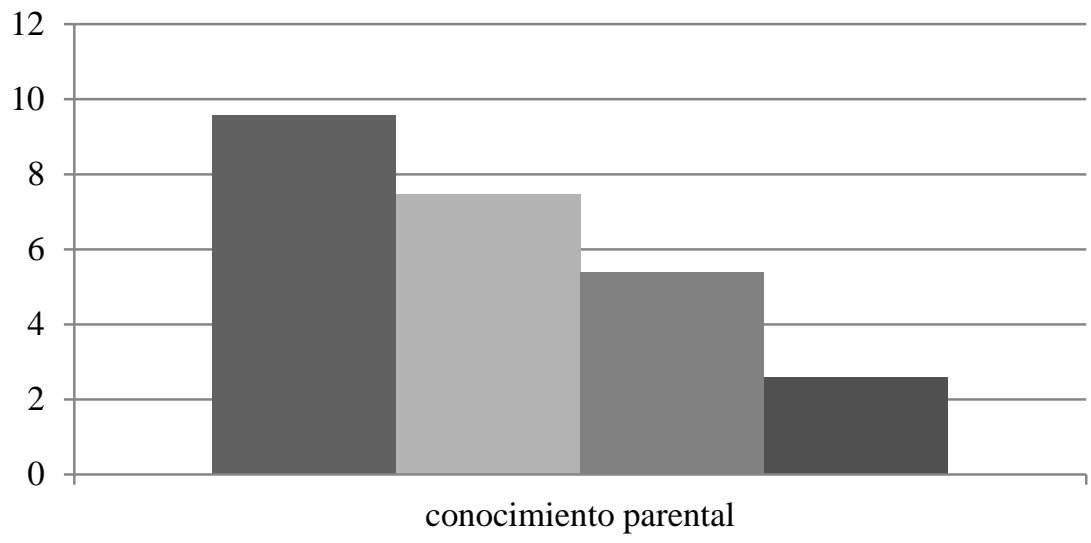

- bajo madre-bajo padre

bajo madre-alto padre

- alto madre-bajo padre

alto madre-alto padre

Figura 3. Consumo de cannabis según el conocimiento parental

\section{Bienestar psicológico}

En la Tabla 5 se observan los valores medios de la calidad de vida relacionada con la salud y la satisfacción vital en función del afecto parental, la promoción parental de la autonomía, el interés parental, la revelación adolescente y el conocimiento parental. En general, los resultados mostraron mayores niveles de calidad de vida relacionada con la salud y satisfacción vital cuando ambos progenitores puntuaron alto en las distintas dimensiones familiares, lo contrario sucedió cuando ambos progenitores puntuaron bajo.

Tabla 5. Datos descriptivos de la calidad de vida relacionada con la salud y la satisfacción vital según las dimensiones familiares

\begin{tabular}{|c|c|c|c|c|c|c|c|}
\hline \multirow{2}{*}{ Variable } & & \multicolumn{3}{|c|}{ Calidad de vida } & \multicolumn{3}{|c|}{ Satisfacción vital } \\
\hline & & $\bar{N}$ & $M$ & $D T$ & $N$ & $M$ & $D T$ \\
\hline \multirow{4}{*}{ Afecto parental } & bajo madre-bajo padre & 836 & 42.07 & 10.53 & 836 & 6.40 & 1.91 \\
\hline & bajo madre-alto padre & 345 & 44.28 & 9.25 & 345 & 6.94 & 1.84 \\
\hline & alto madre-bajo padre & 1651 & 46.65 & 9.74 & 1651 & 7.12 & 1.65 \\
\hline & alto madre-alto padre & 7337 & 51.93 & 9.26 & 7337 & 7.85 & 1.46 \\
\hline \multirow{4}{*}{ Promoción parental de la autonomía } & bajo madre-bajo padre & 2251 & 46.75 & 10.56 & 2251 & 7.25 & 1.85 \\
\hline & bajo madre-alto padre & 1392 & 48.00 & 9.73 & 1392 & 7.20 & 1.64 \\
\hline & alto madre-bajo padre & 1028 & 48.63 & 9.71 & 1028 & 7.33 & 1.65 \\
\hline & alto madre-alto padre & 5499 & 52.09 & 9.36 & 5499 & 7.86 & 1.43 \\
\hline \multirow{4}{*}{ Interés parental } & bajo madre-bajo padre & 1242 & 47.55 & 11.09 & 1242 & 7.17 & 1.96 \\
\hline & bajo madre-alto padre & 484 & 48.99 & 10.13 & 484 & 7.35 & 1.71 \\
\hline & alto madre-bajo padre & 2489 & 47.88 & 9.59 & 2489 & 7.36 & 1.58 \\
\hline & alto madre-alto padre & 5955 & 51.48 & 9.64 & 5955 & 7.78 & 1.51 \\
\hline \multirow{4}{*}{ Revelación adolescente } & bajo madre-bajo padre & 3781 & 48.10 & 10.14 & 3781 & 7.32 & 1.75 \\
\hline & bajo madre-alto padre & 750 & 49.47 & 9.56 & 750 & 7.53 & 1.54 \\
\hline & alto madre-bajo padre & 1901 & 48.33 & 9.50 & 1901 & 7.41 & 1.58 \\
\hline & alto madre-alto padre & 3738 & 52.88 & 9.51 & 3738 & 7.94 & 1.43 \\
\hline \multirow{4}{*}{ Conocimiento parental } & bajo madre-bajo padre & 655 & 44.51 & 11.45 & 655 & 6.77 & 2.09 \\
\hline & bajo madre-alto padre & 69 & 45.46 & 9.66 & 69 & 7.02 & 1.96 \\
\hline & alto madre-bajo padre & 1647 & 46.13 & 9.86 & 1647 & 7.03 & 1.79 \\
\hline & alto madre-alto padre & 7799 & 51.32 & 9.51 & 7799 & 7.77 & 1.47 \\
\hline
\end{tabular}


En cuanto a la calidad de vida relacionada con la salud, en la Tabla 6 se observa que el modelo fue significativo y con tamaño de efecto medio, $F(15,9361)=113.770, p<$ $.001, R^{2}=.154$. El interés parental fue significativo, $F(3$, $9361)=15.469, p<.001$, pero con tamaño de efecto despreciable $\left(\eta^{2}\right.$ parcial $\left.=.005\right)$, al igual que el conocimiento parental, $F(3,9361)=20.124, p<.001, \eta^{2}$ parcial $=.006$. Sin em- bargo, el afecto parental, $F(3,9361)=139.161, p<.001$, la promoción parental de la autonomía, $F(3,9361)=59.031, p$ $<.001$, y la revelación adolescente, $F(3,9361)=36.650, p<$ .001 , fueron significativos, con tamaños de efecto bajomedio para afecto $\left(\eta^{2}\right.$ parcial $\left.=.043\right)$ y bajos para promoción de la autonomía $\left(\eta^{2}\right.$ parcial $\left.=.019\right)$ y para revelación $\left(\eta^{2}\right.$ parcial $=.012)$.

Tabla 6. Modelo lineal general de las dimensiones familiares sobre la calidad de vida relacionada con la salud

\begin{tabular}{|c|c|c|c|c|c|c|}
\hline Fuente & $S C$ & $g l$ & $M C$ & $F$ & $p$ & $\eta^{2}$ parcial \\
\hline Modelo corregido & 142711.503 & 15 & 9514.100 & 113.770 & .000 & .154 \\
\hline Intersección & 1435871.529 & 1 & 1435871.529 & 17170.207 & .000 & .647 \\
\hline Afecto parental & 34912.362 & 3 & 11637.454 & 139.161 & .000 & .043 \\
\hline Promoción parental de la autonomía & 14809.643 & 3 & 4936.548 & 59.031 & .000 & .019 \\
\hline Interés parental & 3880.921 & 3 & 1293.640 & 15.469 & .000 & .005 \\
\hline Revelación adolescente & 9194.587 & 3 & 3064.862 & 36.650 & .000 & .012 \\
\hline Conocimiento parental & 5048.772 & 3 & 1682.924 & 20.124 & .000 & .006 \\
\hline Error & 782820.674 & 9361 & 83.626 & & & \\
\hline Total & 24380000 & 9377 & & & & \\
\hline Total corregida & 925532.176 & 9376 & & & & \\
\hline
\end{tabular}

En relación con el afecto parental, los adolescentes que percibieron muy afectuosos a ambos progenitores puntuaron más alto en calidad de vida que los adolescentes que percibieron poco afectuosos a ambos progenitores $(p<.001, d=$ 1.05) o solo a la madre $(p<.001, d=0.83)$, ambas diferencias con efectos grandes, y que los que percibieron poco afectuoso al padre con un efecto medio $(p<.001, d=0.56)$. Los adolescentes con ambos progenitores con bajos niveles de esta dimensión tuvieron menor calidad de vida que aquellos en los que la madre $(p=.005, d=0.22)$ o el padre fueron poco afectuosos $(\phi<.001, d=0.46)$; y los adolescentes con madres poco y padres muy afectuosos puntuaron más bajo en calidad de vida que los adolescentes en los que sucedió lo contrario $(p<.001, d=0.25)$.
En cuanto a la promoción de la autonomía, cuando ambos progenitores fueron muy promotores tuvieron adolescentes con mayor calidad de vida que los adolescentes cuyos progenitores puntuaron bajo $(p<.001, d=0.55)$ con un efecto medio, o bien que la madre $(p<.001, d=0.43)$ o que el padre puntuaron bajo $(\phi<.001, d=0.37)$.

Finalmente, los adolescentes que revelaron mucha información a ambos progenitores tuvieron mayor calidad de vida relacionada con la salud que los adolescentes que revelaron poca información a ambos progenitores $(p<.001, d=$ $0.49)$, a la madre $(p<.001, d=0.36)$ o al padre $(p<.001, d=$ 0.48). Todas estas diferencias son mostradas en la Figura 4.

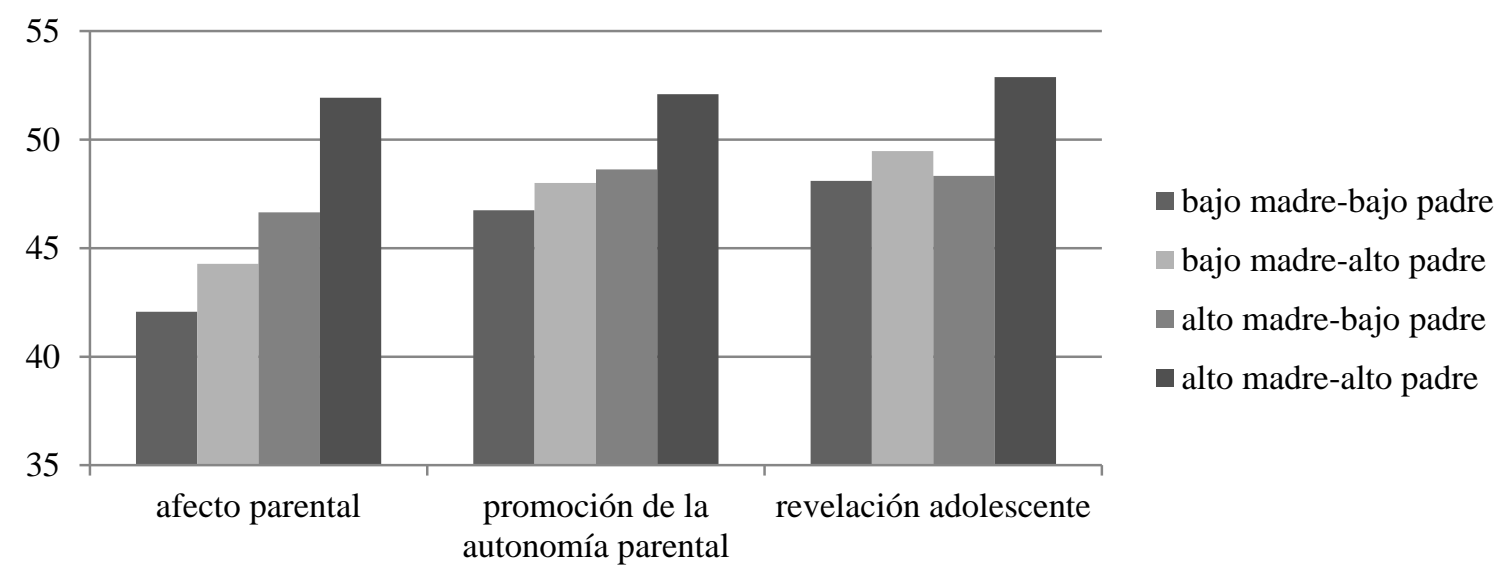

Figura 4. Calidad de vida relacionada con la salud según el afecto parental, la promoción parental de la autonomía y la revelación adolescente

El modelo para la satisfacción vital, como se muestra en la Tabla 7, fue significativo y con tamaño de efecto bajomedio, $F(15,9361)=78.639, p<.001, R^{2}=.112$. A pesar de que fueron significativas la promoción parental de la au- tonomía, $F(3,9361)=29.104, p<.001$, el interés parental, $F$ $(3,9361)=9.974, p<.001$, la revelación adolescente, $F(3$, 9361) $=15.009, p<.001$, y el conocimiento parental, $F(3$, $9361)=20.673, p<.001$, los tamaños de efecto fueron des- 
preciables $\left(\eta^{2}\right.$ parcial $=.009$ para promoción de la autonomía, $\eta^{2}$ parcial $=.003$ para interés, $\eta^{2}$ parcial $=.005$ para revelación y $\eta^{2}$ parcial $=.007$ para conocimiento). Solo fue sig- nificativo, $F(3,9361)=107.847, p<.001$, y con tamaño de efecto bajo $\left(\eta^{2}\right.$ parcial $\left.=.033\right)$ el afecto parental.

Tabla 7. Modelo lineal general de las dimensiones familiares sobre la satisfacción vital

\begin{tabular}{|c|c|c|c|c|c|c|}
\hline Fuente & $S C$ & $g l$ & $M C$ & $F$ & $p$ & $\eta^{2}$ parcial \\
\hline Modelo corregido & 2710.803 & 15 & 180.720 & 78.639 & .000 & .112 \\
\hline Intersección & 33710.019 & 1 & 33710.019 & 14668.591 & .000 & .610 \\
\hline Afecto parental & 743.535 & 3 & 247.845 & 107.847 & .000 & .033 \\
\hline Promoción parental de la autonomía & 200.651 & 3 & 66.884 & 29.104 & .000 & .009 \\
\hline Interés parental & 68.763 & 3 & 22.921 & 9.974 & .000 & .003 \\
\hline Revelación adolescente & 103.475 & 3 & 34.492 & 15.009 & .000 & .005 \\
\hline Conocimiento parental & 142.526 & 3 & 47.509 & 20.673 & .000 & .007 \\
\hline Error & 21512.596 & 9361 & 2.298 & & & \\
\hline Total & 563801.000 & 9377 & & & & \\
\hline Total corregida & 24223.399 & 9376 & & & & \\
\hline
\end{tabular}

Como se observa en la Figura 5, los adolescentes que percibieron a sus padres y madres como muy afectuosos tuvieron mayor satisfacción vital que los adolescentes que percibieron poco afectuosos a sus padres y madres, con una magnitud del efecto grande $(p<.001, d=0.96)$, que los adolescentes con madres con nivel bajo de esta dimensión $(p<$ $.001, d=0.62)$ con efecto medio, y que los adolescentes con padres poco afectuosos $(p<.001, d=0.49)$ con efecto pequeño. Además, cuando el padre $(p<.001, d=0.29)$ o la madre fue percibido muy afectuoso $(p<.001, d=0.41)$, los adolescentes estuvieron más satisfechos con sus vidas que los adolescentes con ambos progenitores con bajos niveles de afecto.

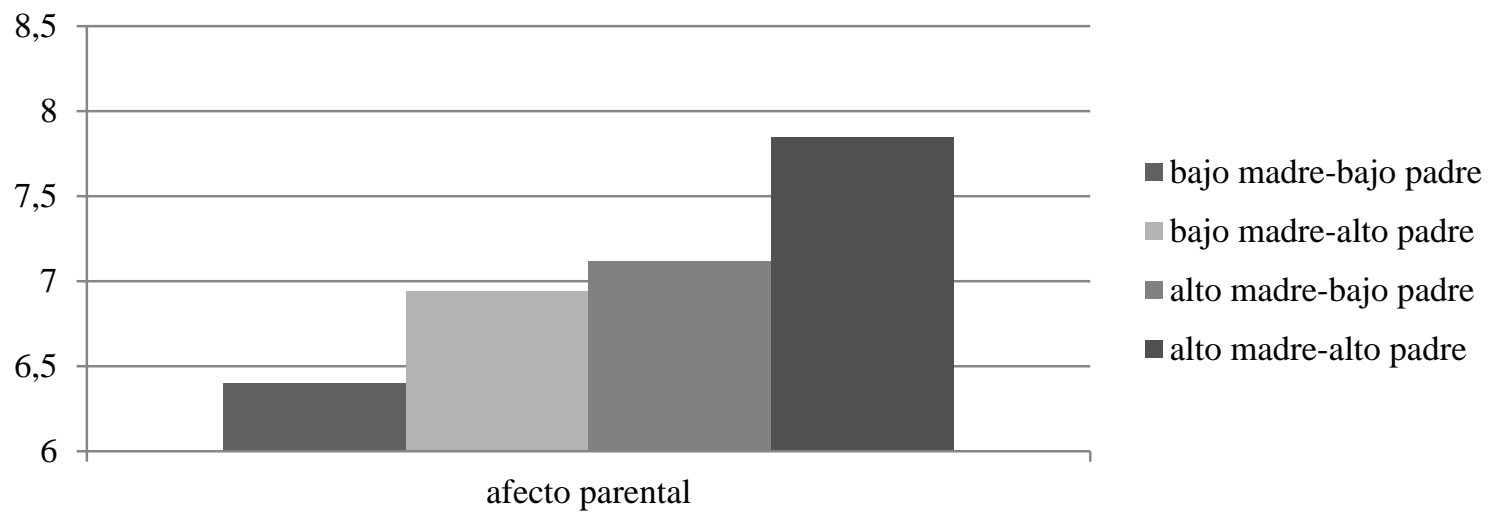

Figura 5. Satisfacción vital según el afecto parental

\section{Discusión}

El objetivo de este trabajo es analizar, desde un enfoque dimensional, cómo influye en el ajuste de los adolescentes, concretamente en su consumo de sustancias y bienestar psicológico, que sus padres y madres presenten ambos puntuaciones altas, bajas o diverjan en las distintas dimensiones familiares analizadas: afecto, promoción de la autonomía, interés, revelación y conocimiento, que son componentes importantes del estilo democrático en la adolescencia.

El estilo parental consistente en padres y madres es más efectivo y deseable que un estilo inconsistente (Dwairy, 2010), con la excepción de que los dos progenitores sean indiferentes, ya que supondrá los peores resultados para los chicos y las chicas (Simons y Conger, 2007). Los mejores resultados en chicas y chicos se encuentran con los dos proge- nitores democráticos (McKinney y Renk, 2008; Simons y Conger, 2007; Steinberg, 2001).

En esta investigación, los resultados más positivos para el ajuste están en los adolescentes con padres y madres percibidos con niveles altos en las distintas dimensiones familiares que reflejan de alguna forma el estilo democrático. Así, los adolescentes con padres y madres muy afectuosos tienen mejor calidad de vida y mayor satisfacción vital que los demás, pero especialmente que los que tienen ambos progenitores poco afectuosos. Igualmente, los chicos y las chicas con ambos progenitores muy promotores de su autonomía presentan mayor calidad de vida, en comparación con el resto, sobre todo, con los adolescentes con ambos progenitores poco dados a estimular su autonomía. De forma similar, cuando revelan mucha información a ambos progenitores presentan mayor calidad de vida que el resto de adolescentes, así como 
menos consumo de alcohol que los adolescentes con baja revelación a ambos progenitores y baja solo a las madres. Finalmente, los chicos y las chicas que perciben a sus padres y madres muy conocedores de sus experiencias consumen menos tabaco que aquellos con ambos progenitores o solo con el padre poco conocedores; también consumen menos alcohol y cannabis que el resto de adolescentes, sobre todo, que los que tienen ambos progenitores con bajo conocimiento y, además, en el caso del consumo de alcohol, que los adolescentes con la madre poco conocedora y el padre muy conocedor.

Como se ha podido apreciar, los adolescentes con madres y padres con puntuaciones bajas en las dimensiones familiares analizadas, lo que vendría a parecerse al estilo indiferente, presentan los peores resultados en ajuste de esta investigación. Otras diferencias distintas a las anteriores corroboran el peor ajuste en los adolescentes con ambos progenitores puntuados bajos en las dimensiones familiares analizadas. Los chicos y las chicas con padres y madres poco afectuosos presentan menor calidad de vida y satisfacción vital que los que tienen, al menos, un progenitor muy afectuoso. Del mismo modo, los adolescentes que perciben a sus padres y madres con bajo conocimiento consumen más tabaco que los que tienen solo un progenitor con bajo conocimiento.

Estos resultados indican que la coherencia entre las prácticas y los estilos educativos de los progenitores no es tan fundamental como tener, al menos, un progenitor democrático (Fletcher et al., 1999; McKinney y Renk, 2008; Steinberg, 2001). En el caso de esta investigación, lo anterior se traduce en un padre o una madre que exprese las dimensiones familiares analizadas, ya que ello puede amortiguar las consecuencias perjudiciales para el ajuste adolescente de no tener progenitores con características parentales positivas (Simons y Conger, 2007; Steinberg, 2001). Por ejemplo, en el caso de un estudio sobre facilidad de comunicación y consumo de sustancias, la comunicación fácil con ambos progenitores favoreció un menor consumo de sustancias, seguido de la comunicación fácil solo con la madre (Moreno et al., 2006).

Otras diferencias significativas señalan una influencia más positiva de las dimensiones maternas que de las paternas. En las familias en las que los adolescentes solo tienen a su madre como muy conocedora y padre poco conocedor consumen menos alcohol y cannabis que los adolescentes que perciben a sus padres y madres con bajo conocimiento. Además, los adolescentes con madres muy afectuosas y padres poco afectuosos tienen mejor calidad de vida que los que están en la situación inversa; y los adolescentes con madres muy conocedoras y padres poco conocedores consumen menos alcohol que los que perciben a sus padres con alto y sus madres con bajo conocimiento. Tal vez esto es debido a que aunque ambos progenitores son importantes para hijos e hijas, la influencia materna tiende a ser algo superior a la paterna (Hair et al., 2008; Rodrigo et al., 2004). Por ejemplo, es más perjudicial para el ajuste adolescente que las madres muestren un estilo indiferente a que lo hagan los padres
(Simons y Conger, 2007), quizá porque para el bienestar adolescente son centrales el afecto materno (Cheng y Furnham, 2004), la comunicación con la madre (Morrow, 2004) y la calidad de la relación con la madre (Trzcinski y Holst, 2008). Tampoco hay que olvidar en esta consideración que la mayor dedicación e influencia de la madre frente al padre responde mejor a las expectativas que nuestra cultura tiene puestas en ambos (Menéndez y Hidalgo, 2003; Menéndez, Hidalgo, Jiménez y Moreno, 2011).

Por último, los datos de este trabajo indican qué dimensiones familiares son más relevantes para el consumo de sustancias y el bienestar psicológico. El conocimiento parental resultó esencial para el consumo de sustancias, como otros estudios han encontrado (Jiménez-Iglesias et al., 2012; Jiménez-Iglesias, Moreno, Rivera y García-Moya, 2013; Soenens, Vansteenkiste, Luyckx y Goossens, 2006; Tebes et al., 2011), dado que permite a los padres y las madres estar informados sobre la vida de sus hijos e hijas y hacer algo con ese conocimiento para proteger a sus adolescentes (Stattin et al., 2010), en una etapa en la que la supervisión parental directa disminuye y las oportunidades para participar en actividades problemáticas aumentan (Jacobson y Crockett, 2000). Por su parte, el afecto parental, dimensión decisiva en el ajuste emocional adolescente (Kagitçibasi, 1996), fue fundamental para el bienestar psicológico, como la calidad de vida y la satisfacción vital (Gillison et al., 2008; Jiménez-Iglesias et al., 2012; Jiménez-Iglesias, Moreno, Ramos y Rivera, 2014; Morgan, Rivera, Moreno y Haglund, 2012; Oliva, Parra, SánchezQueija y López, 2007; Parra y Oliva, 2006).

La revelación adolescente fue importante para el consumo de alcohol y la calidad de vida. En cuanto al consumo de alcohol, probablemente porque si los adolescentes revelan información a sus progenitores, éstos tendrán mayor confianza en que sus hijos e hijas toman buenas decisiones durante el tiempo que no están con ellos (Kerr, Stattin y Trost, 1999) y podrán guiarles para evitar que se impliquen en conductas de riesgo (Marshall, Tilton-Weaver y Bosdet, 2005); por tanto, mayor revelación supondrá menor probabilidad de consumir alcohol (Ghandour, 2009; Jiménez-Iglesias et al., 2012; Jiménez-Iglesias et al., 2013; Stavrinides, Georgiou y Demetriou, 2010). Respecto a la calidad de vida relacionada con la salud, la revelación junto con el interés parental reflejan la comunicación parento-filial (Keijsers et al., 2010), que es protectora del bienestar psicológico, especialmente la comunicación entre progenitores y adolescentes en torno a asuntos que les preocupan (Jiménez-Iglesias et al., 2012; Morrow, 2004; Oliva et al., 2007).

Y la promoción parental de la autonomía resultó relevante para la calidad de vida; los progenitores que son sensibles a las necesidades de individualidad de sus hijos e hijas y les otorgan mayor autonomía, contribuyen a mejorar el bienestar general adolescente (Gillison et al., 2008; Jiménez-Iglesias et al., 2014; Lekes et al., 2010; Smetana et al., 2004).

Entre las limitaciones encontradas en este trabajo están las referidas al hecho de que toda la información proceda de la misma fuente, el chico o la chica adolescente, y se prescin- 
da de la información de madres y padres; a pesar de ello, conviene no olvidar que los adolescentes son la fuente de información más objetiva y menos influida por la deseabilidad social (Gonzales, Cauce y Mason, 1996), así como que sus ideas sobre la adolescencia son más positivas y coinciden con los resultados de investigaciones recientes (Ridao y Moreno, 2008). Otra limitación se debe al diseño de esta investigación de carácter transversal, de validez más limitada que un diseño de tipo longitudinal.

No obstante, es de especial relevancia que este trabajo se dedique a evaluar las diferencias en las distintas dimensiones entre padres y madres, ya que este tema ha recibido poca atención (McKinney y Renk, 2008), lo que ha imposibilitado conocer las consecuencias reales para los hijos y las hijas de las diferencias entre los estilos maternos y los estilos paternos (Simons y Conger, 2007).

\section{Conclusiones}

Como síntesis de los resultados, se puede afirmar que, en general, los adolescentes con padres y madres con altos niveles en las dimensiones familiares analizadas consumen menos sustancias y presentan mejor bienestar psicológico. Por el contrario, en general, los adolescentes con padres y madres con bajos niveles de las dimensiones familiares analizadas presentan mayor consumo y más pobre bienestar. En una si-

\section{Referencias}

Bronstein, P., Duncan, P., D'Ari, A., Pieniadz, J., Fitzgerald, M., Abrams, C. L.,... Oh Cha, S. Y. (1996). Family and parenting behaviors predicting middle school adjustment: A longitudinal study. Family Relations, 45, 415-426. Doi: 10.2307/585171

Brown, B. B., Mounts, N., Lamborn, S. D. y Steinberg, L. (1993). Parenting practices and peer group affiliation in adolescence. Child Development, 64, 467-482. Doi: 10.1111/j.1467-8624.1993.tb02922.x

Bumpus, M. F. y Rodgers, K. B. (2009). Parental knowledge and its sources examining the moderating roles of family structure and race. Journal of Family Issues, 30, 1356-1378. Doi: 10.1177/0192513X09334154

Cantril, H. (1965). The pattern of human concern. Nueva Jersey, EE.UU.: Rutgers University Press.

Casullo, M. M. y Liporace, M. F. (2008). Percepción sobre estilos e inconsistencia parentales en adolescentes argentinos. Estudios de Psicologia (Campinas), 25, 3-9. Doi: 10.1590/S0103-166X2008000100001

Cheng, H. y Furnham, A. (2004). Perceived parental rearing style, selfesteem and self-criticism as predictors of happiness. Journal of Happiness Studies, 5, 1-21. Doi: 10.1023/B:JOHS.0000021704.35267.05

Cohen, J. (1988). Statistical power analysis for the behavioral science. Hillsdale, EE.UU.: Lawrence Erlbaum Associates.

Currie, C., Nic Gabhainn, S., Godeau, E., Roberts, C., Smith, R., Currie, D.,... Barkenow, V. (2008). Inequalities in young people's health: HBSC international report from the 2005/06 Survey. Health policy for children and adolescents (Informe núm. 5). Copenhague, Dinamarca: WHO Regional Office for Europe.

Dwairy, M. (2010). Parental inconsistency: A third cross-cultural research on parenting and psychological adjustment of children. Journal of Child and Family Studies, 19, 23-29. Doi: 10.1007/s10826-009-9339-x

Elliot, D. S. (1993). Health-enhancing and health-compromising lifestyles. En S. G. Millstein, A. C. Petersen y E. O. Nightingale (Eds.), Promoting the health of adolescents: New directions for the twenty-first century (pp.119-150). Nueva York, EE.UU.: Oxford University Press. tuación intermedia se encuentran los adolescentes con al menos un progenitor con altos niveles de las dimensiones familiares. Además, otras diferencias entre padres y madres señalan a las madres como algo más relevantes para el ajuste adolescente.

Los resultados de este trabajo pueden resultar útiles para intervenciones con padres y madres, en el sentido de que indican que ambos progenitores son importantes para los adolescentes y deben esforzarse por actuar adecuadamente en su labor como padres y madres, a ser posible de forma consistente porque cuando padres y madres muestran niveles altos de las mismas dimensiones familiares promueven el mejor ajuste adolescente. Por lo demás, este estudio indica qué dimensiones son necesarias de ser promovidas para un consumo de sustancias más responsable y un mejor bienestar psicológico entre los adolescentes: el conocimiento parental es fundamental para el consumo de sustancias, así como la revelación adolescente para el consumo de alcohol; mientras que el afecto parental es esencial para el bienestar psicológico junto con la promoción parental de la autonomía y la revelación adolescente, específicamente para la calidad de vida relacionada con la salud.

Agradecimientos.- Esta investigación ha sido financiada gracias a un convenio firmado entre el Ministerio de Sanidad, Política Social e Igualdad y la Universidad de Sevilla (España).
Fletcher, A. C., Steinberg, L. y Sellers, E. B. (1999). Adolescents' well-being as a function of perceived interparental consistency. Journal of Marriage and the Family, 61, 599-610. Doi: 10.2307/353563

Gamble, W. C., Ramakumar, S. y Diaz, A. (2007). Maternal and paternal similarities and differences in parenting: An examination of MexicanAmerican parents of young children. Early Childhood Research Quarterly, 22, 72-88. Doi: 10.1016/j.ecresq.2006.11.004

Ghandour, L. A. (2009). Young adult alcohol involvement: The role of parental monitoring, child disclosure, and parental knowledge during childhood. Dissertation Abstracts International: Sección B. Sciences and Engineering, 69(12), 7391.

Gillison, F., Standage, M. y Skevington, S. (2008). Changes in quality of life and psychological need satisfaction following the transition to secondary school. British Journal of Educational Psychology, 78, 149-162. Doi: 10.1348/000709907X209863

Goldstein, S. E., Davis-Kean, P. E. y Eccles, J. S. (2005). Parents, peers, and problem behavior: A longitudinal investigation of the impact of relationship perceptions and characteristics on the development of adolescent problem behavior. Developmental Psychology, 41, 401-413. Doi: 10.1037/0012-1649.41.2.401

Gonzales, N. A., Cauce, A. M. y Mason, C. A. (1996). Interobserver agreement in the assessment of parental behavior and parent-adolescent conflict: African American mothers, daughters, and independent observers. Child Development, 67, 1483-1498. Doi: 10.1111/j.14678624.1996.tb01809.x

Hair, E. C., Moore, K. A., Garrett, S. B., Ling, T. y Cleveland, K. (2008). The continued importance of quality parent-adolescent relationships during late adolescence. Journal of Research on Adolescence, 18, 187-200. Doi: 10.1111/j.1532-7795.2008.00556.x

Hibell, B., Andersson, B., Ahlström, S., Balakireva, O., Bjarnasson, T., Kokkevi, A. y Morgan, M. (2000). The 1999 European School Survey Project on Alcohol and Other Drugs (ESPAD) Report. Alcohol and other drug use among students in 30 European countries. Estocolmo, Suecia: The Swedish 
Council for Information on Alcohol and Other Drugs (CAN) and The Pompidou Group at the Council of Europe.

Hublet, A. y Godeau, E. (2005). HBSC Research Protocol for 2005/06 Survey. Section 2. Scientific rationales for focus areas: Tobacco use. Manuscrito inédito.

Jacobson, K. C. y Crockett, L. J. (2000). Parental monitoring and adolescent adjustment: An ecological perspective. Journal of Research on Adolescence, 10, 65-97. Doi: 10.1207/SJRA1001_4

Jiménez-Iglesias, A., Moreno, C., Granado-Alcón, M. C. y López, A. (2012). Parental knowledge and adolescent adjustment (substance use and Health-Related Quality of Life). The Spanish Journal of Psychology, 15, 132144. Doi: $10.5209 /$ rev_SJOP.2012.v15.n1.37297

Jiménez-Iglesias, A., Moreno, C., Ramos, P. y Rivera, F. (2014). What family dimensions are important for health-related quality of life in adolescence? Journal of Youth Studies. Publicación anticipada en línea. Doi: 10.1080/13676261.2014.933191

Jiménez-Iglesias, A., Moreno, C., Rivera, F., y García-Moya, I. (2013).The role of the family in promoting responsible substance use in adolescence. Journal of Child and Family Studies, 22, 585-602. Doi: 10.1007/s10826-013-9737-y

Kagitçibasi, C. (1996). Family and human development across countries: A view from the other side. Nueva Jersey, EE.UU.: Lawrence Erlbaum Associates.

Keijsers, L., Branje, S. J. T., VanderValk, I. E. y Meeus, W. (2010). Reciprocal effects between parental solicitation, parental control, adolescent disclosure, and adolescent delinquency. Journal of Research on Adolescence, 20, 88-113. Doi:10.1111/j.1532-7795.2009.00631.x.

Kerr, M., Stattin, H. y Trost, K. (1999). To know you is to trust you: Parents' trust is rooted in child disclosure of information. Journal of Adolescence, 22, 737-752. Doi: 10.1006/jado.1999.0266

Klimidis, S., Minas, I. H. y Ata, A. W. (1992). The PBI-BC: A brief current form of the parental bonding instrument for adolescent research. Comprehensive Psychiatry, 33, 374-377. Doi: 10.1016/0010-440X(92)90058-X

Lekes, N., Gingras, I., Philippe, F. L., Koestner, R. y Fang, J. (2010). Parental autonomy-support, intrinsic life goals, and well-being among adolescents in China and North America. Journal of Youth and Adolescence, 39, 858-869. Doi: 10.1007/s10964-009-9451-7

Marshall, S. K., Tilton-Weaver, L. C. y Bosdet, L. (2005). Information management: Considering adolescents' regulation of parental knowledge. Journal of Adolescence, 28, 633-647. Doi: 10.1016/j.adolescence.2005.08.008

McKinney, C. y Renk, K. (2008). Differential parenting between mothers and fathers: Implications for late adolescents. Journal of Family Issues, 29, 806-827. Doi: 10.1177/0192513X07311222

Menéndez, S. y Hidalgo, M. V. (2003). La evaluación de varones y mujeres de sus papeles como cónyuges y como padres y madres: análisis de las relaciones entre ambos roles. Anuario de Psicología, 34, 81-99. Recuperado de http://www.raco.cat/index.php/anuariopsicologia

Menéndez, S., Hidalgo, M. V., Jiménez, L. y Moreno, C. (2011). Father involvement and marital relationship during transition to parenthood in Spain: Differences between dual- and single-earner families. The Spanish Journal of Psychology, 14, 639-647. Doi: 10.5209/rev_SJOP.2011.v14.n2.12

Moreno, C., Muñoz-Tinoco, V., Pérez Moreno, P. J. y Sánchez-Queija, I. (2006). Los adolescentes españoles y sus familias: calidad en la comunicación con el padre y con la madre y conductas de riesgo relacionadas con el consumo de sustancias adictivas. Cultura y Educación, 18, 345-362. Doi: $10.1174 / 113564006779172975$

Moreno, C., Ramos, P., Rivera, F., Muñoz-Tinoco, V., Sánchez-Queija, I., Granado, M. C. y Jiménez-Iglesias, A. (2011). Desarrollo adolescente y salud en España. Resumen del estudio Health Behaviour in School-aged Children (HBSC-2006). Madrid: Ministerio de Sanidad, Política Social e Igualdad.

Morgan, A., Rivera, F., Moreno, C. y Haglund, B. (2012). Does social capital travel? Influences on the life satisfaction of young people living in England and Spain. BMC Public Health, 12:138. Doi:10.1186/1471-2458-12138

Morrow, V. (2004). Children's social capital: Implications for health and well-being. Health Education, 104, 211-225. Doi: 10.1108/09654280410546718
Oliva, A., Parra, Á., Sánchez-Queija, I. y López, F. (2007). Estilos educativos materno y paterno: Evaluación y relación con el ajuste adolescente. Anales de Psicología, 23, 49-56.

Parra, Á. y Oliva, A. (2006). Un análisis longitudinal sobre las dimensiones relevantes del estilo parental durante la adolescencia. Infancia y Aprendiraje, 29, 453-470. Doi: 10.1174/021037006778849594

Reitz, E., Deković, M. y Meijer, A. M. (2006). Relations between parenting and externalizing and internalizing problem behaviour in early adolescence: Child behaviour as moderator and predictor. Journal of Adolescence, 29, 419-436. Doi: 10.1016/j.adolescence.2005.08.003

Ridao, P. y Moreno, C. (2008). Percepción de los adolescentes y sus progenitores de la adolescencia como etapa evolutiva. Infancia y Aprendiraje, 31, 499-513. Doi: 10.1174/021037008786140904

Rodrigo, M. J., Máiquez, M. L., García, M., Mendoza, R., Rubio, A., Martínez, A. y Martín, J. C. (2004). Relaciones padres-hijos y estilos de vida en la adolescencia. Psicothema, 16, 203-210. Recuperado de http://www.psicothema.com/

Schmid, H., Fotiou, A., Godeau, E., Simons-Morton, B. y Hublet, A. (2005). HBSC Researcb Protocol for 2005/06 Survey. Section 2. Scientific rationales for focus areas: Alcohol use. Manuscrito inédito.

Shaughnessy, J. J., Zechmeister, E. B. y Zechmeister, J. S. (2007). Métodos de investigación en psicologia ( $7^{a}$ ed.). Madrid, España: McGraw-Hill.

Simons, L. G. y Conger, R. D. (2007). Linking mother-father differences in parenting to a typology of family parenting styles and adolescent outcomes. Journal of Family Issues, 28, 212-241. Doi: 10.1177/0192513X06294593

Smetana, J. G., Campione-Barr, N. y Daddis, C. (2004). Longitudinal development of family decision making: Defining healthy behavioral autonomy for middle-class African American adolescents. Child Development, 75, 1418-1434. Doi: 10.1111/j.1467-8624.2004.00749.x

Soenens, B., Vansteenkiste, M., Luyckx, K. y Goossens, L. (2006). Parenting and adolescent problem behavior: An integrated model with adolescent self-disclosure and perceived parental knowledge as intervening variables. Developmental Psychology, 42, 305-318. Doi: 10.1037/00121649.42.2.305

Stattin, H., Kerr, M. y Tilton-Weaver, L. (2010). Parental monitoring: A critical examination of the research. En V. Guilamo-Ramos, J. Jaccard y P. Dittus (Eds.), Parental Monitoring of Adolescents: Pt. 1. Contemporary issues in parental monitoring (pp. 3-38). Nueva York, EE.UU.: Columbia University Press.

Stavrinides, P., Georgiou, S. y Demetriou, A. (2010). Longitudinal associations between adolescent alcohol use and parents' sources of knowledge. British Journal of Developmental Psychology, 28, 643-655. Doi: 10.1348/026151009X466578

Steinberg, L. (2001). We know some things: Parent-adolescent relationships in retrospect and prospect. Journal of Research on Adolescence, 11, 1-19. Doi: $10.1111 / 1532-7795.00001$

Steinberg, L. y Silk, J. S. (2002). Parenting adolescents. En M. H. Bornstein (Ed.), Handbook of Parenting: Vol. 1. Children and parenting ( $2^{\mathrm{a}}$ ed., pp. 103134). Nueva Jersey, EE.UU.: Lawrence Erlbaum Associates.

Tebes, J. K., Cook, E. C., Vanderploeg, J. J., Feinn, R., Chinman, M. J., Shepard, J. K.,... Connell, C. M. (2011). Parental knowledge and substance use among African American adolescents: Influence of gender and grade level. Journal of Child and Family Studies, 20, 406-413. Doi: 10.1007/s10826-010-9406-3

The KIDSCREEN Group Europe (2006). The KIDSCREEN questionnaires. Quality of life questionnaires for children and adolescents. Handbook. Lengerich, Alemania: Pabst Science.

Trzcinski, E. y Holst, E. (2008). Subjective well-being among young people in transition to adulthood. Social Indicators Research, 87, 83-109. Doi: 10.1007/s11205-007-9160-0

Williams, P. G., Holmbeck, G. N. y Neff Greenley, R. (2002). Adolescent health psychology. Journal of Consulting and Clinical Psychology, 70, 828-842. Doi: $10.1037 / / 0022-006 X .70 .3 .828$

(Articulo recibido: 15-9-2012; revisado: 17-5-2013; aceptado: 20-2-2014) 
